GA-A23978

\title{
RADIAL TRANSPORT EFFECTS ON ECCD IN THE TCV AND DIII-D TOKAMAKS AND ON OHMIC DISCHARGES IN THE MST RFP
}

by
R.W. HARVEY, O. SAUTER, R. PRATER, P. NIKKOLA, R. O'CONNELL,
and C.B. FOREST 


\section{DISCLAIMER}

This report was prepared as an account of work sponsored by an agency of the United States Government. Neither the United States Government nor any agency thereof, nor any of their employees, makes any warranty, express or implied, or assumes any legal liability or responsibility for the accuracy, completeness, or usefulness of any information, apparatus, product, or process disclosed, or represents that its use would not infringe privately owned rights. Reference herein to any specific commercial product, process, or service by trade name, trademark, manufacturer, or otherwise, does not necessarily constitute or imply its endorsement, recommendation, or favoring by the United States Government or any agency thereof. The views and opinions of authors expressed herein do not necessarily state or reflect those of the United States Government or any agency thereof. 
GA-A23978

\title{
RADIAL TRANSPORT EFFECTS ON ECCD IN THE TCV AND DIII-D TOKAMAKS AND ON OHMIC DISCHARGES IN THE MST RFP
}

\author{
by \\ R.W. HARVEY,† O. SAUTER, ‡ R. PRATER, P. NIKKOLA, ‡ R. O’CONNELL,, \\ and C.B. FOREST $\diamond$ \\ †CompX \\ ҒEcole Polytechnique Fédérale de Lausanne \\ $\checkmark$ University of Wisconsin, Madison
}

This is a preprint of a paper presented at the 12th Joint Workshop on Electron Cyclotron Emission and Electron Cyclotron Resonance Heating, May 13-16, 2002, in Aix-en-Provence, France, and to be published in the Proceedings.

\section{Work supported by the U.S. Department of Energy \\ under Contract DE-AC03-99ER54463, and Grants DE-FG03-99ER54541 and DE-FG03-96ER54373}




\title{
RADIAL TRANSPORT EFFECTS ON ECCD IN THE TCV AND DIII-D TOKAMAKS AND ON OHMIC DISCHARGES IN THE MST RFP
}

\author{
R.W. Harvey, ${ }^{1}$ O. Sauter, ${ }^{2}$ R. Prater, ${ }^{3}$ P. Nikkola, ${ }^{2}$ R. O’Connell, ${ }^{4}$ and C.B. Forest ${ }^{4}$ \\ ${ }^{1}$ CompX, P.O. Box 2672, Del Mar, California $92014-5672$ \\ ${ }^{2}$ Ecole Polytechniuque Fédérale de Lausanne, CH-1015, Lausanne, Switzerland \\ ${ }^{3}$ General Atomics, P.O. Box 85608, San Diego, California 92186-5608 \\ ${ }^{4}$ University of Wisconsin, Madison, Wisconsin 53706-1687
}

\begin{abstract}
The comprehensive CQL3D Fokker-Planck/Quasilinear simulation code has been benchmarked against experiment over a wide range of electron cyclotron conditions in the DIII-D tokamak (C.C. Petty et al., 14th Topical Conf. on RF Power in Plasmas, 2002). The same code, in disagreement with experiment, gives $560 \mathrm{kA}$ of ECCD for a well documented, completely ECCD-driven, $100 \mathrm{kA}$ TCV shot [O. Sauter et al, PRL, 2000]. Recent work (R.W. Harvey et al, Phys. Rev. Lett., 2002) has resolved the differences as due to radial transport at a level closely consistent with ITER scaling. Transport does not substantially affect DIII-D ECCD, but at similar ECH power has an overwhelming effect on the much smaller TCV. The transport is consistent with electrostatic-type diffusion $\left(\mathrm{D}_{\rho} \rho\right.$ constant in velocityspace) and not with a magnetic-type diffusion $\left(D_{\rho \rho} \propto|v| \mid\right)$.

Fokker-Planck simulation of Ohmic reversed field pinch (RFP) discharges in the MST device reveals transport velocity dependence stronger than $|\mathrm{v} \||$ will give agreement with current and soft $\mathrm{X}$-ray spectra in standard discharges, but in the higher confinement, current profile controlled PPCD discharges, transport is again electrostatic-like. This is consistent with the object of PPCD, which is to replace magnetic turbulence driven current with auxiliary CD to improve transport. The tokamak and high-confinement RFP results mutually reinforce the constant-in-velocity-space "electrostatic-type turbulence" conclusion. The steady-state energy and toroidal current are governed by the same radial transport equation.
\end{abstract}

The role of radial transport on high energy electrons in tokamaks and reversed field pinches (RFPs), particularly in regards to radiofrequency (rf) experiments, has been examined by several authors [1-8, and references therein]. At high enough power, the plasma temperature is high and the transport effects strong enough such that radial transport dominates the collisional slowing down time of the fast electrons. Our calculations [8] show that this is strongly the case for a representative full-toroidal-current-drive electron cyclotron current drive (ECCD) experiment [9] in the TCV fusion energy tokamak. Although there is little doubt as to whether plasma turbulence is responsible for the observed radial transport in excess of collisional levels [10], questions remain on the extent electrostatic (ES) or magnetic turbulence dominates [11] and also the degree of concurrence between tail electron transport and bulk plasma transport. In tokamaks our work shows good agreement between experiment and modeling based on radial diffusion due to ES turbulence at a level predicted by wellknown empirical modeling [10]; it shows poor agreement with the purely magnetic turbulence model.

In the MST reversed field pinch (RFP) device at UW, Madison, a similar result has been found: In standard Ohmic MST discharges the transport appears to be due to magnetic-type turbulence; however, when the plasma current driven by magnetic turbulence in the outer part of the plasma is replaced by transiently driven Ohmic pulsed poloidal current (PPCD), the transport improves and switches from magnetic-type to being dominated by electrostatictype [12].

The primary results for DIII-D and TCV have been reported in Ref. 8. In this paper we report on further aspects of the calculation, and outline some of the new, related results being obtained in the MST device.

The calculations are performed with the comprehensive CQL3D FokkerPlanck/Quasilinear (FP/QL) simulation code [4]. The FP model includes two-dimensional in momentum-space collisional diffusion, the full Stix [13] rf QL diffusion coefficient, a radial diffusion $\mathrm{D}_{\rho \rho}$ and pinch term in non-circular flux-surface geometry, and is relativistic. The pinch term is adjusted to maintain a target density profile. Steady-state, finite-difference numerical solutions are obtained for the electron distribution $f_{\mathrm{e}}\left(\mathrm{u}_{0}, \theta_{0}, \rho\right)$, evaluated at the outer equatorial plane of the toroidal plasma, where $\mathrm{u}_{0}=\mathrm{p} / \mathrm{m}_{\mathrm{e}}$ is momentum-per-electron-rest- 
mass, $\theta_{0}$ is momentum-space pitch angle, and $\rho$ is a generalized radial coordinate labeling the toroidal flux surfaces. For the TCV modeling, the velocity dependence of $\mathrm{D}_{\rho \rho}$ is chosen to be either constant (ES turbulence-type) or proportional to the magnitude of the electron velocity $\mid \mathrm{v} \| / \mathrm{v}_{\mathrm{T}_{\mathrm{e}}}$ parallel to the ambient magnetic field (magnetic turbulence type) divided by the thermal velocity $\mathrm{v}_{\mathrm{e}}=\left(\mathrm{T}_{\mathrm{e}}(\rho) / \mathrm{m}_{\mathrm{e}}\right)^{1 / 2}$. The radial dependence of $\mathrm{D}_{\rho \rho}$ is chosen to increase towards the plasma periphery, $\mathrm{D}_{\rho \rho}=\mathrm{D}_{\rho \rho 0}\left(1+3(\rho / \mathrm{a})^{3}\right)\left(\mathrm{n}_{\mathrm{e} 0} / \mathrm{n}_{\mathrm{e}}(\rho)\right)$ in general accord with experimental observations for the relevant low confinement L-mode [14]. The EC radiation field is obtained from data coupled into CQL3D from the TORAY-GA [15] ray tracing code. We expect that, using the experimentally measured profiles of plasma density $\left(\mathrm{n}_{\mathrm{e}}\right)$, temperature $\left(\mathrm{T}_{\mathrm{e}}\right)$, and effective charge $\mathrm{Z}_{\mathrm{eff}}$, the CQL3D facility can provide a full and accurate physics-based model of the ECCD process in tokamaks, apart from the radial transport effects which are to be discerned by comparison with experiment.

The CQL3D code is run with collisions of the electron distribution occurring on ions and on a self-consistent distribution of the electrons for which the angular dependence is expanded in Legendre polynomials, except that the coefficient of the $\mathrm{P}_{0}\left(\theta_{0}\right)$ term is maintained at the Maxwellian corresponding to the radially local experimental temperature. This has the effect of maintaining the local bulk of the electron distribution as a Maxwellian with the experimental parameters; the tail electrons then collisionally interact with the dominant bulk and radially diffuse. Momentum is conserved in the electron-electron interaction, preserving accurate determination of the parallel current.

To perform an accurate comparison between radial diffusion of the tail particles and a given (ITER) confinement scaling [10], it is necessary to account for the 2-D, non-circular effects. These are treated in a manner consistent with the ONETWO transport code [16].

We consider the radial transport of the quantity $\lambda f / \oint\left(\mathrm{dl}_{\mathrm{B}} / \psi\right)$ which is the flux surface volume-average of particles in the momentum-space element $\mathrm{d}^{3} \mathbf{u}_{0}$. Quantity $\psi=\mathrm{B} / \mathrm{B}_{0}$, and $l_{B}$ is length along $\mathbf{B} ; B$ is magnetic field strength and $B_{0}$ its minimum value on a flux surface. The radial operator conserves flux surface averaged density $\langle n\rangle=\int \mathrm{d}^{3} \mathbf{u}_{0} \lambda f / \oint\left(\mathrm{dl}_{\mathrm{B}} / \psi\right)$. For noncircular geometry, we obtain ${ }^{4}$

$$
\begin{aligned}
\frac{\partial(\lambda \mathrm{f})}{\partial \mathrm{t}}= & \langle\langle\mathrm{R}\rangle\rangle+\ldots=\left.\left|\frac{\mathbf{u}_{\| 0}}{\mathrm{~B}_{0}}\right|^{\mathrm{C}_{0}} \frac{\oint \mathrm{dl}_{\mathrm{B}} / \psi}{\mathrm{H} \rho} \frac{\partial}{\partial \rho}\right|_{\mathrm{C} 1, \mathrm{C} 2} \mathrm{H} \rho\left|\frac{\mathrm{B}_{0}}{\mathbf{u}_{\| 0}}\right|^{\mathrm{C}_{0}} \\
& \cdot\left\{\left.\mathrm{D}_{\rho \rho} \frac{\partial}{\partial \rho}\right|_{\mathrm{C} 1, \mathrm{C} 2}\left[\frac{\lambda f}{\oint\left(\mathrm{dl}_{\mathrm{B}} / \psi\right)} \mathrm{V}_{\mathrm{p}} \frac{\lambda f}{\oint\left(\mathrm{dl}_{\mathrm{B}} / \psi\right)}\right]\right\}
\end{aligned}
$$

where

$$
\left(\mathrm{C}_{0}, \mathrm{C}_{1}, \mathrm{C}_{2}\right)=\left\{\begin{array}{c}
(1, \mathrm{E}, \mathrm{u}) \text { constant } \mathrm{E}, \mathrm{u} \text { diffusion } \\
\left(0, \mu_{0}, \theta_{0}\right) \text { constant } \mathrm{u}_{0}, \theta_{0} \text { diffusion }
\end{array}\right.
$$

$\mathrm{H}$ is a quantity previously used in the ONETWO transport code [16] containing the effects of noncircular geometry, and is equal to 1 , for circular concentric flux surfaces. The quantity $\mathrm{u}_{\| 0} / \mathrm{B}_{0}$ appears in the Jacobian of the transformation from $\mathrm{u}_{0}, \theta_{0^{-}}$to $\mathrm{E}, \mu$-coordinates, $\mathrm{d}^{3} \mathbf{u}_{0}=\left|\mathrm{B}_{0} / \mathbf{u}_{\| 0}\right|\left(2 \pi \gamma / \mathrm{m}^{2}\right) \mathrm{dEdu}$ The radial diffusion coefficient $\mathrm{D}_{\rho \rho}$ and the advective pinch term $V_{\rho}$ are arbitrary functions of velocity and radius. The above radial transport equation is in a conservative form. In the present work, we have used "constant $\mathrm{u}_{0}, \theta_{0}$-diffusion," although "constant $E, \mu$-diffusion is appropriate for turbulence below the cyclotron frequency. Taking momentum-space moments of the above radial transport operator gives the radial transport terms in the ONETWO code.

The agreement between DIII-D tokamak EC experiments and the CQL3D code has been excellent over a wide range of EC experiments and injection conditions [17], neglecting radial transport effects. The experiments discussed here on TCV and DIII-D are very similar: plasma densities and temperatures and magnetic field strength are almost the same; approximately $1.5 \mathrm{MW}$ of X-mode EC radiation is injected from the off-midplane outboard side of the tokamak to resonate with plasma electrons near the second harmonic of the cyclotron frequency. The major difference between the two tokamaks is that DIII-D is 2.5 
times greater in each linear dimension, or approximately 15 times the volume of TCV. Hence EC power density in the TCV tokamak is overwhelmingly stronger than in DIII-D.

A key point in regard to the TCV and DIII-D tokamaks is that they both provide data for the ITER empirical transport studies [10] and are consistent with the same transport scaling laws as fit the data from the other nine tokamaks in the data base. Thus, we may expect that transport in these devices is governed by the same general turbulence processes.

We examine the TCV shot 16099 which is fully supported by EC current drive [9]. The EC ray geometry is shown superimposed on a cross-section of the toroidal flux surfaces in Fig. 1. Spreading the 1.5 MW of $\mathrm{EC}$ heating and $\mathrm{CD}$ as shown in the figure results in a stable discharge for the $2.0 \mathrm{~s}$ duration of the available EC power [9].

In the absence of radial transport, the calculated ECCD in TCV is $550 \mathrm{kA}$, more than 5 times the experimentally measured value. The effect of radial transport in reduction of current drive efficiency in the very high power EC experiment in TCV is dramatic. ITER L-mode scaling [10] applied to TCV shot 16099 gives confinement time $2.5 \mathrm{~ms}$, as compared to the experimentally determined value $2.1 \mathrm{~ms}$ [9]. Transforming this to an estimate of the radial diffusion coefficient $\mathrm{D}_{\rho \rho 0}=0.5 * \mathrm{a}^{2} / 4 \tau_{\mathrm{E} \text {, th }}^{\mathrm{L}}$ (the " 0.5 " accounts approximately for the radial dependence of $\mathrm{D} \rho \rho$ gives $\mathrm{D} \rho \rho_{0}=3.0 \mathrm{~m}^{2} \mathrm{~s}$. The value of $\mathrm{D} \rho \rho_{0}$ necessary in order to fit the observed EC driven current obtained for the electrostatic-type diffusion is $D \rho \rho_{0} \approx 3.7 \mathrm{~m}^{2} \mathrm{~s}$, whereas for the magnetic-type diffusion the value is $0.35 \mathrm{~m}^{2} / \mathrm{s}$.

If the magnetic turbulence were increased sufficiently to fit the bulk electrons, it would be a factor of 10 too strong for the code to match the observed ECCD. Thus, the results reported here provide strong support for ES-type turbulence, not purely magnetic turbulence, dominating both the bulk and the tail electron transport. On the other hand, there is the possibility that a more complicated velocity dependence of $D_{\rho \rho}$ will give the same good fit to the data as has been reported here. This will be the subject of further studies.

We now provide further detail on the simulation of TCV. At the top of Fig. 2 are shown cuts at constant pitch angle through the electron distribution for plasma radius $0.1 \mathrm{a}$, and below them are the corresponding specific current density $\mathrm{j}_{\mu}(\mathrm{u})$ versus $\mathrm{u} / \mathrm{v}_{\mathrm{T}_{\mathrm{e}}}$, where current density $\mathrm{j}=\int \mathrm{j}_{\mathrm{u}}(\mathrm{u}) \mathrm{du}$. The case (a) at the left of the figure is with no radial diffusion, and the case (b) at the right is with ES-type radial diffusion coefficient $3.7 \mathrm{~m}^{2} / \mathrm{s}$. The lower-u portion of the distribution remains Maxwellian at the given experimental temperature. Without radial diffusion a large current carrying tail distribution is formed out to near the edge of the velocity-mesh.

The average electron energy versus radius is shown in Fig. 3. In the absence of transport, huge average energy is carried by the nonthermal tail. With transport, values
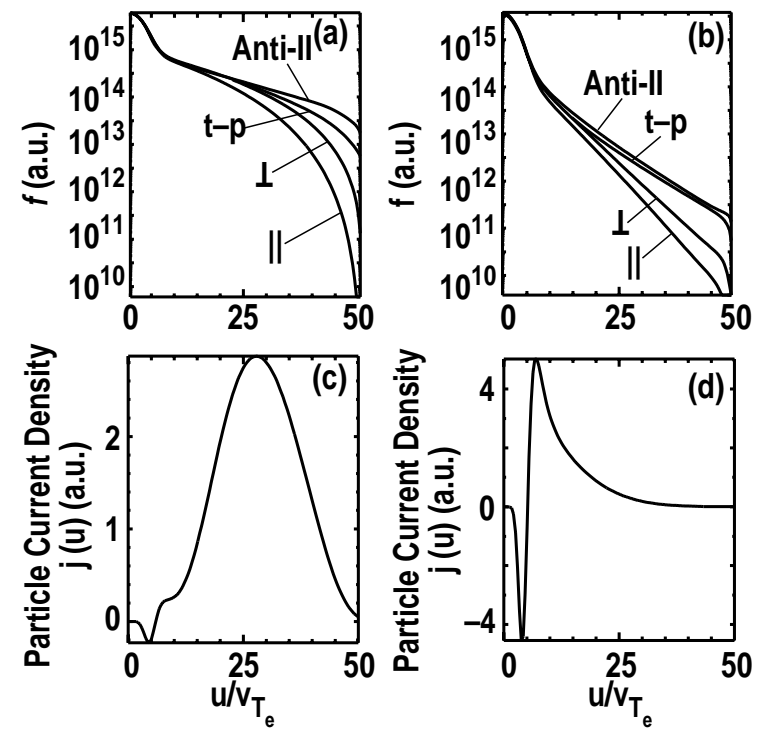

Fig. 2. Left column (a) shows cuts through the distribution as a function of $u$, at various pitch angles; below is the distribution $j_{u}(u)$ of driven current, resulting from ECCD in TCV with no radial diffusion. The right column $(b)$ is the same, but calculated with radial diffusion turned on. 
compatible with the experiment are obtained. In the figures, $\mathrm{D}_{\mathrm{rr} 0}$ is the same as $\mathrm{D}_{\rho \rho 0}$.

Transport sharply reduces the tail electron current to the $100 \mathrm{kA}$ experimental value, as shown in Fig. 4. This current is slightly above the $82 \mathrm{kA}$ obtained linearly in CQL3D by evaluating CD at a very low power in the absence of $D_{\rho \rho}$ and renormalizing back to the experimental power. The CQL3D linear value is slightly greater than the $70 \mathrm{kA}$ from the TORAY code, based on the Cohen [18] model (which neglects current excited in the thermal portion of the electron distribution by the electron-electron collisions). The central value of current density $j(\rho=0)$ has been reduced to a value giving safety factor $q$ $=1.0$, in agreement with the marginal MHD stability of this shot [9].

The DIII-D data [17] was modeled under the condition that radial transport is turned off in the code. In fact, a scan of ES $D_{\rho \rho 0}$ from 0 to $4.0 \mathrm{~m}^{2} / \mathrm{s}$ gives the result that the rf driven current (including synergy with the induced toroidal electric field) only reduces from $45.1 \mathrm{kA}$ to $40.0 \mathrm{kA}$, for a standard benchmark shot (\#104017). This change in current is within the experimental error bars. The value $D_{\rho \rho 0}=1.0 \mathrm{~m}^{2} / \mathrm{s}$ is obtained from the ITER L-mode $\tau_{\mathrm{E}, \mathrm{th}}^{\mathrm{L}}$-scaling for this shot, as previously discussed. The resulting calculated radial current density profiles are shown in Fig. 5. Thus, although the DIII-D analysis for CD efficiency which is the focus of comparison with CQL3D thus far, is not appreciably changed, there is an important spreading of the driven EC current. This effect may need to be considered for accurate calculation of EC stabilization of neo-classical tearing modes [9-22].

The standard RFP relies on transport and dynamo effects of overlapping islands associated with neoclassical tearing modes to flatten its current profile, maintaining a stable $q<1$ equilibria. Numerical studies suggested that these modes could be stabilized by flattening the current profile by driving current in the edge, thus improving confinement. This has been done on the Madison symmetric torus (MST) by the application of PPCD. The mode amplitudes fall dramatically and there is a corresponding order of magnitude increase in the confinement time to $10 \mathrm{~ms}$ and hard Xrays (HXRs) are seen for the first time in an RFP.

Given the basic plasma profiles, CQL3D has been used to model the PPCD period of these plasmas. Density, temperature, and $\mathrm{Z}_{\mathrm{eff}}$ (in the PPCD discharges) are measured. $\mathrm{E}_{\phi}$ and $\mathrm{j} \|$ are determined from the timedependent equilibrium reconstructions. In the FP code, the applied electric field is iterated until the driven current matches the measured equilibrium current. The diffusion coefficient is left as a free variable as a function of space and energy. Best agreement with predicted CQL3D X-ray flux is for a diffusion

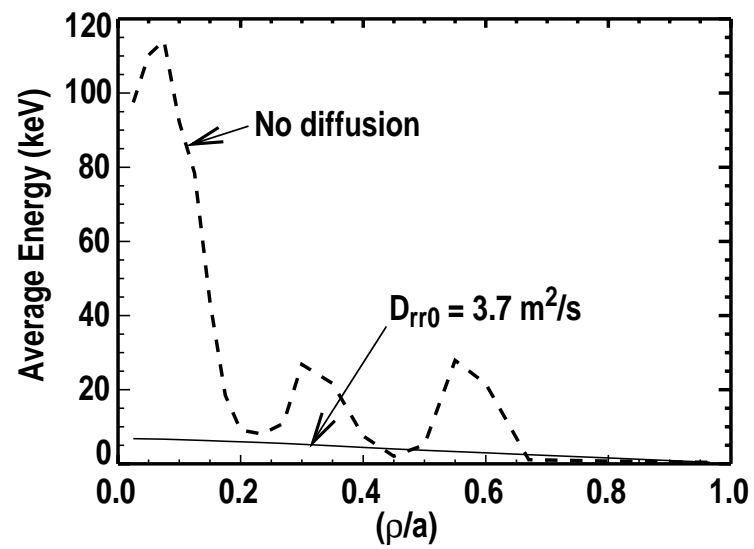

Fig. 3. Average plasma energy in TCV, calculated without and with radial diffusion.

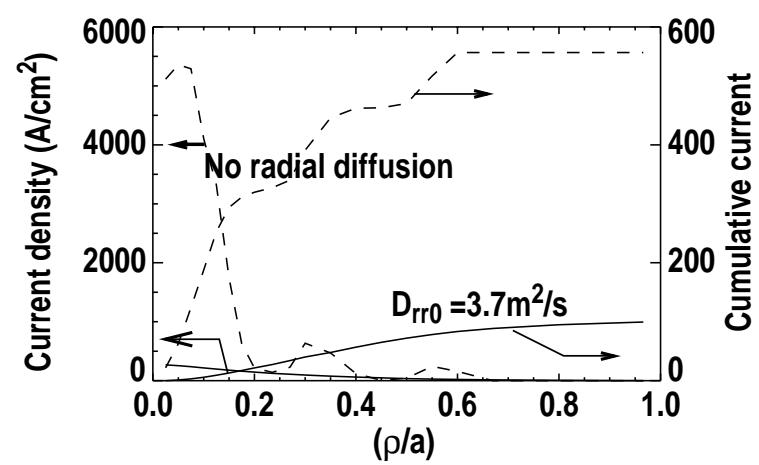

Fig. 4. Radial profile of current density in TCV, calculated without and with radial diffusion.

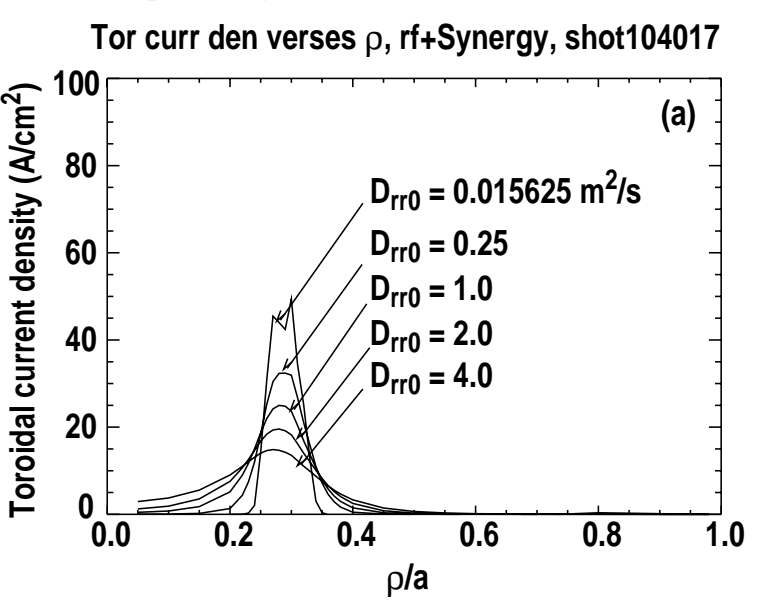

Fig. 5. Calculated radial profile of current density in the DIII-D experiment, shot 105017, for various levels of radial diffusion. $D_{r r O}=1.0 \mathrm{~m}^{2} / \mathrm{s}$ matches the ITER L-mode scaling. 
coefficient of $\sim 1 \mathrm{~m}^{2} / \mathrm{s}$ independent of velocity (see Fig. 6). The plasma resistivity, which is a good indicator of the bulk electron behavior is also best fit by a diffusion coefficient of $\sim 1 \mathrm{~m}^{2} / \mathrm{s}$, as shown in Fig. 7 . This suggests that the transport is no longer dominated by stochastic magnetic diffusion, rather electrostatic diffusion.

In conclusion, we have shown that, for the TCV and DIII-D EC tokamak experiments which span a substantial range of the ITER transport scaling data base, the calculation of the radial transport effects at levels predicted by the ITER data base maintains agreement between experimental observations of ECCD and accepted ECCD physics. In TCV, the transport effect is overwhelming, and the agreement with transport scaling laws is accurate. Electrostatic-type turbulence is implied, and not purely magnetic-type turbulence. In DIII-D, as the EC system power is increased transport will also become more prominent. The X-ray signals indicate that, in the improved PPCD regime of the RFP, the MST device is also achieving a regime of transport dominated by electrostatic turbulence.

\section{Acknowledgment}

Work supported by U.S. Department of Energy under Contract DE-AC03-99ER54463 and Grants DE-FG03-99ER54541 and DE-FG03-96ER54373.

\section{References}

[1] M.G. McCoy and R.W. Harvey, "Effects of Anomalous Transport on LH Electron Heating," Proc. 4th Topical Conf. on RF Plasma Heating, C7, R.D. Bengtson and M.E. Oakes Eds., UT, Austin (1981).

[2] B. Lloyd et al., Nucl. Fusion 28, 1013 (1988).

[3] R.W. Harvey, M.G. McCoy, and G.D. Kerbel, Phys. Rev. Lett. 62, 426 (1989).

[4] R.W. Harvey and M.G. McCoy, Proc. of IAEA TCM on Numerical Modeling of Plasmas, Montreal, pp. 489-526, 1992 (IAEA, Vienna, 1993).

[5] Y. Peysson, Plasma Phys. Control. Fusion 35, B253 (1993).

[6] G. Giruzzi and E. Martines, Phys. Plasmas 1, 2653 (1994); G. Giruzzi et al., Phys. Rev. Lett. 74, 550 (1995).

[7] A.G. Peters and E. Westerhof, Phys. Plasmas 3, 1628 (1996).

[8] R.W. Harvey, O. Sauter, R. Prater, and P. Nikkola, to be published in Phys. Rev. Lett. 88, No. 20 (2002); see also, P. Nikkola, O. Sauter, et al., this conference.

[9] O. Sauter, et al., Phys. Rev. Lett. 84, 3322 (2000).

[10] ITER Physics Basis, Chapter 2: Plasma confinement and transport, Nucl. Fusion 39, $2175 \mathrm{ff}$ (1999).

[11] J.W. Connor, Plasma Phys. Control. Fusion 35, B293 (1993).

[12] R. O'Connell et al., APS2001.

[13] T. H. Stix, Waves in Plasmas, AIP, N.Y. (1992).

[14] K.H. Burrell et al., in Plasma Phys. and Control. Nucl. Fusion Research, (Proc. 13th Int. Conf., Washington, 1990), Vol. 1, 123 (1991); also, our Ref. [7]; M.R. Wade et al., Phys. Plasmas 2, 2357 (1995). 
[15] K. Matsuda, IEEE Trans. Plasma Sci. 17, 6 (1989); A.H. Kritz et al., Proc. 3rd Joint Varenna-Grenoble International Sym. on Heating in Toroidal Plasmas, Brussels (Oxford NY, 1982), 2, 707.

[16] W.W. Pfeiffer, R.H. Davidson, R.W. Miller, and R.E. Waltz, "ONETWO: A Computer Code for Modeling Plasma Transport in Tokamaks:, General Atomics Report GA-A16178 (1980); F.L. Hinton and R.D. Hazeltine, Rev. Mod. Phys. 48, 239 (1976).

[17] C.C. Petty et al., to be published in AIP Proc. of the 14th Topical Conf. on RF Power in Plasmas, Oxnard, 2001; T.C. Luce et al., Phys. Rev. Lett. 83, 4550 (1999); C.C.Petty, et al., Nucl. Fusion, 35, 773 (1995).

[18] R.H. Cohen, Phys. Fluids 30, 2442 (1987); 31, 421 (1988).

[19] O. Sauter et al., Phys. of Plasmas 4, 1654 (1997).

[20] H. Zohm, Phys. Plasmas 4, 3433 (1997).

[21] R.W. Harvey and F.W. Perkins, Nucl. Fusion 41, 1847 (2001).

[22] R. Prater et al., this conference (2002). 